\title{
Towards quantification of blood-flow changes during cognitive task activation using perfusion-based fMRI
}

\author{
Toralf Mildner, ${ }^{\mathrm{a}, *}$ Stefan Zysset, ${ }^{\mathrm{a}}$ Robert Trampel, ${ }^{\mathrm{a}, \mathrm{b}}$ Wolfgang Driesel, ${ }^{\mathrm{a}}$ and Harald E. Möller ${ }^{\mathrm{a}, \mathrm{c}}$ \\ ${ }^{a}$ Max Planck Institute for Human Cognitive and Brain Sciences, Stephanstr. 1a, 04103 Leipzig, Germany \\ ${ }^{\mathrm{b}}$ Department of Radiology, NYU School of Medicine, New York, NY 10016, USA \\ 'Department of Radiology, University Hospital Münster, Münster, Germany
}

Received 22 December 2004; revised 29 March 2005; accepted 28 April 2005

Available online 22 June 2005

\begin{abstract}
Multi-slice perfusion-based functional magnetic resonance imaging (pfMRI) is demonstrated with a color-word Stroop task as an established cognitive paradigm. Continuous arterial spin labeling (CASL) of the blood in the left common carotid artery was applied for all repetitions of the functional run in a quasi-continuous fashion, i.e., it was interrupted only during image acquisition. For comparison, blood oxygen level dependent (BOLD) contrast was detected using conventional gradient-recalled echo (GE) echo planar imaging (EPI). Positive activations in BOLD imaging appeared in p-fMRI as negative signal changes corresponding to an enhanced transport of inverted water spins into the region of interest, i.e., increased cerebral blood flow (CBF). Regional differences between the localization of activations and the sensitivity of p-fMRI and BOLD-fMRI were observed as, for example, in the inferior frontal sulcus and in the intraparietal sulcus. Quantification of CBF changes during cognitive task activation was performed on a multi-subject basis and yielded CBF increases of the order of $20-30 \%$.
\end{abstract}

(C) 2005 Elsevier Inc. All rights reserved.

Keywords: Arterial spin labeling; Cerebral blood flow; Functional perfusion imaging; Quantification; fMRI; Cognitive task activation

\section{Introduction}

In the last decade, perfusion imaging using magnetic resonance imaging (MRI) techniques with water as an endogenous tracer has been a topic of growing research. From early on, the perfusion contrast created by magnetically labeling of the blood or the tissue was also employed for mapping task-related brain activity (Edelman et al., 1994; Kwong et al., 1992). However, due to its higher sensitivity, the blood oxygen level dependent (BOLD) contrast became the standard tool for functional MRI (fMRI). Despite this fact, interest in perfusion-based fMRI (p-fMRI) remained high.

\footnotetext{
* Corresponding author. Fax: +49 3419940221. E-mail address: mildner@cbs.mpg.de (T. Mildner).

Available online on ScienceDirect (www.sciencedirect.com).
}

Expected improvements in the localization of activation due to the capillary/tissue origin of the perfusion contrast, and in the quantification of functional signal changes were the attracting arguments (Buxton et al., 1998; Siewert et al., 1996; Yang et al., 1998; Ye et al., 1997). Techniques were developed to increase the number of slices, to reduce the transit-time sensitivity, and to improve the time resolution in p-fMRI (Alsop and Detre, 1998; Calamante et al., 1999; Pekar et al., 1996; Zhang et al., 1995). It was shown that p-fMRI has some additional advantages for multisubject studies or at low task frequencies (Wang et al., 2003). However, except for a single-slice study employing a workingmemory task (Ye et al., 1998), all published studies were limited to primary cortical areas, such as the visual or the motor cortex.

The aim of the present work was to detect more subtle functional changes of cerebral blood flow (CBF) related to a cognitive task in different areas of the human brain. For this purpose, a continuous arterial spin labeling (CASL) approach with separate labeling and imaging coils was employed (Zaharchuk et al., 1999). With this technique, all blood entering the brain through one common carotid artery (CCA) is labeled by adiabatic spin inversion. It eliminates unwanted magnetization-transfer effects and allows for an easy implementation of multi-slice perfusion imaging with arbitrary slice orientation. Quantitative mapping of CBF changes in the primary motor cortex by use of this technique was shown recently (Mildner et al., 2003). This study had utilized a post-label delay (PLD) of the order of the maximum transit time to reduce the transit-time dependence of the CASL signal change (Alsop and Detre, 1996). If short PLDs are employed, functional changes of the transit time additionally contribute to the CASL signal change upon stimulation (Gonzalez-At et al., 2000; Hernandez-Garcia et al., 2004). In the latter study, a dual-coil setup with a sampling interval of the order of the arterial transit time was employed to map CBF changes in the primary motor cortex during finger tapping.

The approach used in the current work was designed especially for cognitive p-fMRI studies with the need of acquiring enough slices to cover several cortical areas. A total of eight slices allowed averaging of the single-subject results to achieve sufficient 
sensitivity for the detection of rather subtle CBF changes during cognitive task activation. CASL was applied during all repetitions of the functional run, whereas the acquisition of control images was performed in a short separate scan. An adapted single-trial version of the color-word Stroop interference task (Stroop, 1935) was employed for cognitive task activation. This experiment is known to produce robust activations in the lateral prefrontal, the fronto-median, and parietal cortices and has previously been studied with both gradient-echo (GE) (Zysset et al., 2001) and spin-echo (SE) BOLD-based fMRI (Norris et al., 2002). In the current study, p-fMRI was applied to map and quantify CBF changes in several well-separated brain areas and to compare the results to GE-BOLD-fMRI.

\section{Methods}

MRI procedures

All experiments were performed using a 3-T whole-body scanner (MedSpec 30/100, Bruker BioSpin, Ettlingen, Germany). For image acquisition, a helmet resonator of 24-cm diameter and 13-cm length (Merkle and Driesel, 2003) was used, and for CASL, a circular surface coil of 6-cm diameter was used, both under pulse-program control. The labeling coil was placed over the left CCA of the subject. Active detuning of the radiofrequency (RF) coils was not required because a distance of more than $17 \mathrm{~cm}$ between the center of the labeling coil and the bottom of the helmet resonator was sufficient to guarantee that couplings between both resonance circuits were insignificant. Compared to previous experiments (Mildner et al., 2003), the labeling procedure was slightly altered by increasing the distance between the surface coil and the skin, and by using higher RF labeling power. This leads to an improved homogeneity of the RF magnetic field $B_{1}$ in the region of the CCA without exceeding the local specific absorption rate (SAR). In the present design, this was achieved by a $9-\mathrm{mm}-$ thick insulation of the coil winding. The gradient strength, needed to fulfill the adiabatic condition, was adjusted to $2 \mathrm{mT} \mathrm{m}^{-1}$. The labeling efficiency, $\alpha$, was estimated by simulations of the adiabatic inversion process (Trampel et al., 2004) under consideration of an experimental velocity profile in the CCA recorded for the whole cardiac cycle (Möller et al., 1996). The obtained value of $\alpha \approx 85 \%$ was subsequently used for flow quantification, cf. Appendix A.

During the functional paradigm, labeling was applied quasicontinuously, i.e., interrupted only during the acquisition of images. In particular, control images without labeling were not acquired during the same functional run (Mildner et al., 2003). The labeling power was approximately $1.2 \mathrm{~W}$ applied for the initial 2.5 $\mathrm{s}$ within each repetition time interval of $\mathrm{TR}=3 \mathrm{~s}$. Eight slices were acquired sequentially from superior to inferior $(\mathrm{FOV}=19.2 \mathrm{~cm}$, voxel size $3 \mathrm{~mm} \times 3 \mathrm{~mm} \times 5 \mathrm{~mm}$, interslice distance $6 \mathrm{~mm}$ ) during the final $500 \mathrm{~ms}$ of the TR interval. The slice angulations were adjusted individually for each subject in order to cover the locations of the expected activations (e.g., the inferior frontal junction). For imaging, an SE echo planar imaging (EPI) sequence with a $64 \times 36$ acquisition matrix was used (acquisition bandwidth $100 \mathrm{kHz}$; echo time, $\mathrm{TE}=13 \mathrm{~ms}$; echo position at $11.1 \%$ ). Crusher gradients with a strength of $21 \mathrm{mT} \mathrm{m}^{-1}$, a duration of $1 \mathrm{~ms}$, and a separation of $6.4 \mathrm{~ms}$ were used in order to spoil unwanted magnetization. This corresponds to a $b$ factor of $0.2 \mathrm{~s} \mathrm{~mm}^{-2}$.
Image reconstruction was performed by the half-Fourier reconstruction technique described by Jesmanowicz et al. (1998), which was adapted to a $64 \times 64$ matrix with 4 overscan lines.

Some specific properties of the current CASL implementation which have an impact on the local sensitivity require further consideration: (i) $\mathrm{CBF}$ investigations are limited to the vascular territory of the left CCA due to the position of the labeling coil. (ii) Local differences in the transit time and the presence of intraluminal spins may affect the signal intensity. (iii) Due to a substantial $\mathrm{B}_{1}$ gradient along the symmetry axis of the helmet resonator (Merkle and Driesel, 2003), the signal-to-noise ratio (SNR) is reduced for deeper brain structures, which limits the coverage of the experiment. To account for the combined influence of these effects on the local sensitivity, a baseline experiment consisting of 80 repetitions was recorded in the resting state before the start of the functional paradigm. During this baseline scan, eight repetitions without CASL were followed by eight repetitions with CASL. All imaging and timing parameters were identical to those applied in p-fMRI (see above). The statistical analysis ( $z$ scores) of the baseline scan yielded a map of the sensitivity for each slice, i.e., the extent to which the signal in each voxel was affected by the application of CASL. These maps are subsequently referred to as label-maps and were used as a mask in the further evaluation of the functional experiments.

In addition to $\mathrm{p}$-fMRI, functional control measurements without the application of the labeling RF were performed under otherwise identical conditions using the identical paradigm. These experiments are subsequently referred to as no-label fMRI (nl-fMRI), and were required in order to remove the perturbing influence of BOLD effects (see below).

For comparison to conventional activation maps, GE-BOLDfMRI was performed using a $64 \times 64$ EPI sequence (acquisition bandwidth $100 \mathrm{kHz}$, echo position at $50 \%$, TR = $2 \mathrm{~s}, \mathrm{TE}=30 \mathrm{~ms}$ ). Again, the identical paradigm was used for these experiments.

\section{Subjects}

Written informed consent was obtained from all 16 volunteers (7 male, 9 female, age 22-32 years; mean age 25.5 years) prior to the scanning session. All subjects were right handed, had normal or corrected to normal vision, normal color vision, and were native German speakers. No subject had a history of neurological, major medical, or psychiatric disorders; none was taking medication at the time of measurement.

\section{fMRI procedures}

For task activation, a color-word matching Stroop task (Stroop, 1935; Zysset et al., 2001) was used. The task was presented using a blocked design and has previously been described in detail (Zysset et al., 2001). Briefly, in the Stroop task, the subject has to determine whether the color of symbols presented in the top row matches the color-word in the bottom row (printed in black). Half of the trials resulted in 'YES' responses (right index finger) and the remaining half in 'NO' responses (right middle finger). Visual stimuli were presented with an LCD projector onto a translucent screen positioned inside the magnet bore behind the subject's head. The screen was viewed using a mirror. Responses and reaction times were collected using a keypad. 
In each functional run, 20 blocks including 16 trials of $1.5 \mathrm{~s}$ length, resulting in a block length of $24 \mathrm{~s}$, were presented. Between each block, a resting baseline of $12 \mathrm{~s}$ was introduced. Subjects completed 320 trials per functional run. Eight subjects were observed in a combined session consisting of two functional runs with p-fMRI and another two with nl-fMRI. The order of the functional runs was randomized across the subjects. Standard GEBOLD-fMRI was performed in another eight subjects for comparison.

\section{fMRI data analysis}

The data processing was performed using the software package LIPSIA (Lohmann et al., 2001), which contains tools for preprocessing, co-registration, statistical evaluation, and visualization of fMRI data. Functional data were corrected for motion using a matching metric based on linear correlation. To correct for the temporal offset between the slices acquired in one scan, a cubic-spline interpolation was applied. Further, a temporal highpass filter with a cutoff frequency of $1 / 144 \mathrm{~Hz}$ was used for baseline correction of the signal, and a spatial Gaussian filter with $\alpha=0.8$ (5.65-mm full width at half maximum [FWHM]) was applied.

To align the functional data slices with a 3D stereotactic coordinate reference system, a rigid linear registration with six degrees of freedom (3 rotational, 3 translational) was performed. A $T_{1}$-weighted 3D reference data set (MP-RAGE, cf. Mugler and Brookeman, 1990) had been acquired for each subject during a previous scanning session. This volume data set with an isotropic resolution of $1 \mathrm{~mm}$ was standardized to the Talairach stereotactic space (Talairach and Tournox, 1988). The rotational and translational parameters were obtained on the basis of 2D $T_{1}$-weighted anatomical images, which were aligned with the functional slices and acquired with a modified driven equilibrium Fourier transform (MDEFT) sequence (Norris, 2000). The rotational and translational parameters were subsequently transformed by linear scaling to a standard Talairach size. The resulting parameters were then used to transform the functional slices using trilinear interpolation, so that the resulting functional slices were aligned with the stereotactic coordinate system.
The nl-fMRI time courses of all subjects were group-averaged in the stereotactic coordinate system for each voxel. Before statistical evaluation of each individual p-fMRI data set, the group-averaged nl-fMRI time course was subtracted in each voxel from the p-fMRI time course, in order to remove the remaining BOLD sensitivity of the imaging sequence. The statistical evaluation was based on a least-squares estimation using the general linear model for serially autocorrelated observations (Friston, 1994; Friston et al., 1998). The design matrix was generated with a box-car function, convolved with the hemodynamic response function. The model equation, including the observation data, the design matrix, and the error term, was convolved with a Gaussian kernel of dispersion of 4-s FWHM to deal with the temporal autocorrelation (Worsley and Friston, 1995). In the following, contrast-images between specified conditions were generated for each subject. The contrast between the different conditions was calculated using the $t$ statistic. Subsequently, $t$ values were transformed to $z$ scores. As noted before, each individual functional data set was aligned with the standard stereotactic reference space, so that a group analysis based on the contrastimages could be performed. A group analysis was performed with a Gaussian test by averaging individual $z$ maps and multiplying each $z$ value with the square root of the number of subjects $N$ (Bosch, 2000). The resulting statistical maps were masked with the groupaveraged label-map at a threshold of $P<0.001$, so that for all contrasts, only activations are reported which were affected by CASL. The signal change in the label-map or in the p-fMRI map was obtained by the difference of the averaged signals from label/nolabel periods or Stroop/resting periods, respectively. The CBF change was calculated from these two quantities based on existing theory for the CASL signal change as detailed in Appendix A.

\section{Results}

The top row of Fig. 1 shows sections of the label-map created by the dual-coil CASL experiment averaged over eight subjects. It indicates that perfusion contrast is created mostly in the labeled left hemisphere of the brain with some overflow into the right hemisphere. The label-map indicates a uniform perfusion contrast for the medial and lateral cortices. The sensitivity decreases for regions close to the boundary of the statistically significant
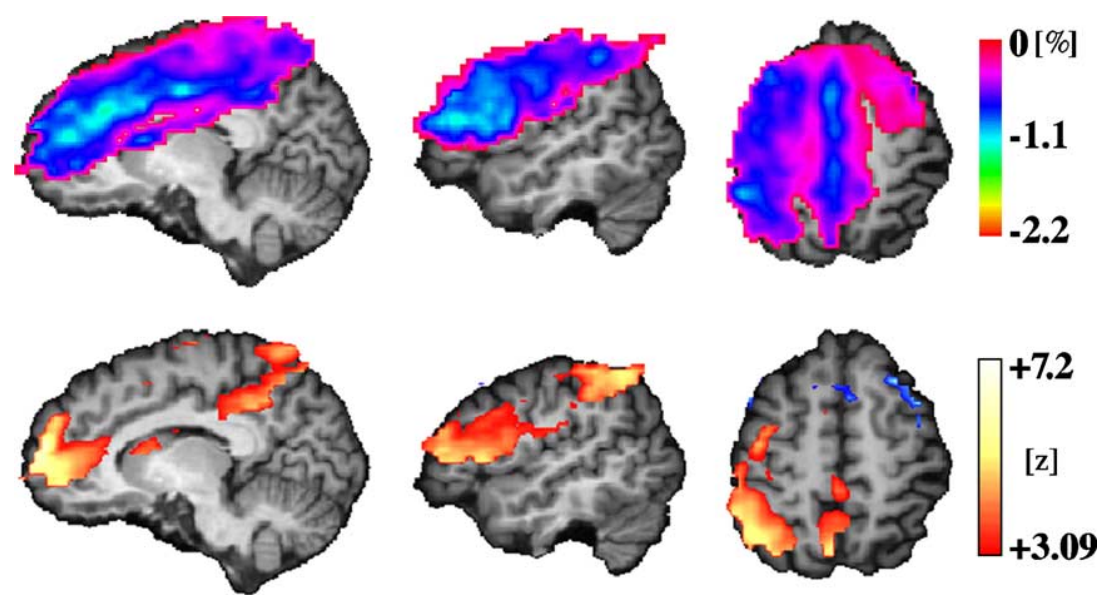

Fig. 1. Group-averaged map of percentage CASL signal change in the resting state (label-map, top) and activation map of nl-fMRI during the Stroop task contrasted against baseline (bottom) and mapped onto an individual brain. Maps are thresholded at $z=3.09(P<0.001 ;$ uncorrected). Shown are views at $x=$ -11 (left), $x=-49$ (middle), and $z=50$ (right). 
regions, especially for regions at the upper edge of the tilted slice package within the parietal cortex. This can be attributed to the slightly different angulations of the slice packages for the individual subjects, which results in less statistical power in brain regions which were not covered in all single-subject experiments. Transit-time differences between the various brain regions may also contribute to this variability.

The bottom row of Fig. 1 shows sections of the nl-fMRI map obtained with the Stroop task. This map indicates that, despite its short TE, the SE-EPI sequence does not suppress the influence of BOLD effects completely. The remaining BOLD sensitivity varies substantially throughout the brain. The strongest nl-fMRI signals were detected in the anterior fronto-median wall, the lateral parietal, and the anterior fronto-lateral cortex.

Fig. 2 shows activation maps obtained for the Stroop task with GE-BOLD-fMRI and with p-fMRI (after the removal of residual BOLD contributions, cf. Methods). Three sections were chosen showing the main activations (i.e., sections through the peaks of the main activations of the p-fMRI map): two sagittal views of the medial cortex and the lateral cortex, and one axial view. GEBOLD signal changes were detected mainly in the left (i.e., the labeled) hemisphere of the brain as expected (Zysset et al., 2001). Table 1 gives coordinates of the most significant activations (positive signal changes with BOLD fMRI and negative signal changes with $\mathrm{p}$-fMRI). These coordinates refer to the local maximum (or minimum) of the corresponding contrast in the given brain area. If no values are given, $\mathrm{p}$-fMRI exhibited no local minimum. The activated areas in p-fMRI are rather small compared to the wide-ranging positive GE-BOLD activations. A good agreement between the localization of activations observed with both contrasts was found for the regions of the presupplementary motor area (pSMA), the primary motor cortex (M1) and, to a lesser extent, in the area of the inferior frontal sulcus (IFS), cf. Fig. 2. However, in the intraparietal sulcus (SIP) and the frontal eye field (FEF), strong GE-BOLD activations were not detectable with p-fMRI.

Time courses of the GE-BOLD- and p-fMRI during a single trial of the Stroop task are given in Fig. 3. A ratio between the temporal contrast-to-noise ratios of p-fMRI and GE-BOLD-fMRI of about 2:7 can be derived. Nevertheless, some features of the GE-BOLD response can be identified in the p-fMRI data, such as an overshoot at the beginning of the Stroop period in M1. Table 1 gives the signal changes for each contrast, i.e., the relative difference of the mean signals during the Stroop task and rest. In the last two columns of Table 1, the CASL signal change in the label-map and the calculated percentage CBF change during the Stroop task are given.

\section{Discussion}

The separate acquisition of the label-map (i.e., of the CASL/ control experiment in the resting state) provides the possibility to assign most of the acquisition time to the mapping of the small functional $\mathrm{CBF}$ changes. The label-map reflects the ability to detect blood-flow changes in different brain regions, because the CASL signal changes upon activation are directly proportional to the CASL signal change in the resting state (Mildner et al., 2003). Consistently, activations in $\mathrm{p}$-fMRI did not reach significance for the areas with the lowest CASL signal change in the label-map (see Table 1). Separate acquisition of the label-map preserves the possibility to quantify $\mathrm{CBF}$ changes. However, a potential source of error might be a change in the baseline CBF between the acquisition of the label-map and the functional part of the session. In our type of experiments or more generally in cognitive studies with multi-subject averaging, this potential error will be reduced by randomly alternating the acquisition of the label-map, acquiring it either at the beginning or at the end of each individual session.

The acquisition of the nl-fMRI map (i.e., a measure of the remaining BOLD sensitivity of the imaging sequence) and the subtraction of the group-averaged nl-fMRI time course from each individual $\mathrm{p}$-fMRI data set were required to improve the detection of small CBF changes due to the Stroop task. BOLD signal changes reduce the sensitivity of p-fMRI, because the corresponding signal changes upon activation are of opposite sign. For our SE-EPI sequence with $\mathrm{TE}=13 \mathrm{~ms}$, the remaining BOLD signals are most likely due to intravascular contributions (Ugurbil et al., 1999), which were not eliminated by the crusher gradients of the sequence.

It is tempting to explain the spatial shifts among activations detected with GE-BOLD-fMRI and p-fMRI in the IFS (Fig. 2;
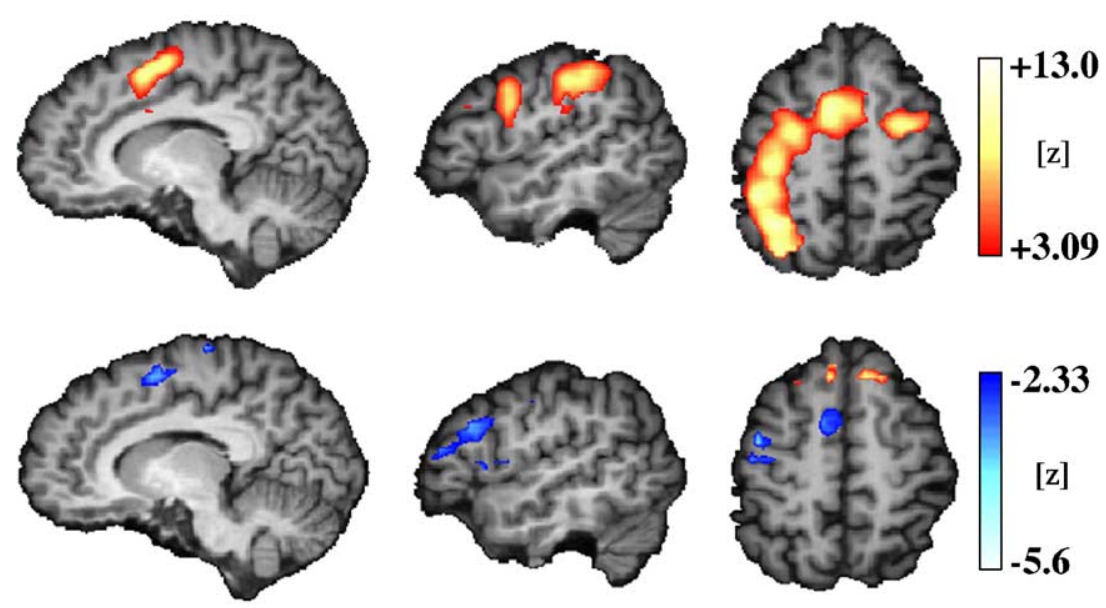

Fig. 2. Activation maps obtained with GE-BOLD-fMRI (top) and p-fMRI (bottom) for the Stroop task contrasted against baseline. The maps represent the average over all subjects and are mapped onto an individual brain. GE-BOLD and p-fMRI maps are thresholded at $z=3.09(P<0.001)$ and $z=2.33(P<$ 0.005 ), respectively. Shown are views at $x=-11$ (left), $x=-49$ (middle), and $z=50$ (right). 
Table 1

Talairach-coordinates at the local maxima of the most prominent activations

\begin{tabular}{|c|c|c|c|c|c|c|c|c|c|c|}
\hline \multirow[t]{2}{*}{ Area } & \multicolumn{3}{|c|}{ GE-BOLD } & \multicolumn{3}{|c|}{ p-fMRI } & \multicolumn{3}{|c|}{ Signal change $[\%]$} & \multirow[t]{2}{*}{$\Delta \mathrm{CBF}[\%]$} \\
\hline & $x$ & $y$ & $z$ & $x$ & $y$ & $z$ & BOLD & $\mathrm{p}$-fMRI & Label-map & \\
\hline FEF & -26 & -9 & 44 & & & & 0.30 & & -0.40 & \\
\hline IFS & -41 & 0 & 30 & -49 & 18 & 29 & 0.63 & -0.18 & -0.87 & 21 \\
\hline M1 & -35 & -25 & 52 & -40 & -19 & 56 & 0.45 & -0.15 & -0.58 & 26 \\
\hline SIP & -41 & -42 & 41 & & & & 0.53 & & -0.55 & \\
\hline pSMA & -7 & 5 & 49 & -10 & 2 & 53 & 0.64 & -0.17 & -0.63 & 27 \\
\hline
\end{tabular}

The last four columns contain the percentage signal changes of GE-BOLD, p-fMRI, and the label-map as well as the percentage CBF change in the given brain areas. In the empty fields, no significant local minimum of p-fMRI was found.

middle column) by different anatomical origins: GE-BOLDfMRI is expected to stem from the venous compartment (Ugurbil et al., 1999), whereas p-fMRI has its origin on the capillary/arterial side of the vascular tree (Calamante et al., 1999). However, we currently cannot exclude the possibility that this spatial shift and the failure to detect p-fMRI activations in the FEF and SIP might still be due to confounding BOLD contributions.

Improvements of the current p-fMRI experiment will be achievable under several aspects. Labeling of both carotid arteries, an improved coverage of the imaging coil, and active detuning of the labeling coil are straightforward steps towards whole-brain coverage (Talagala et al., 2004; Zaharchuk et al., 1999). An even stronger main magnetic field (leading to a prolonged longitudinal relaxation time in the arteries, $T_{1 \mathrm{a}}$ ) would reduce losses of the label during the transit, and thereby, on top of an overall improved SNR of the images, increase the sensitivity. Imaging sequences with even shorter TE's (e.g., by utilizing spiral $k$-space trajectories) or stronger flow-weighting would further decrease the perturbing influence of remaining BOLD signals, which is the main limitation of the current method. This might render the acquisition of nl-fMRI maps dispensable.

In theory, the optimum sensitivity of p-fMRI at given TR (i.e., the maximum CASL signal change) is obtained for truecontinuous labeling (TCL, i.e., labeling even during image acquisition). The CASL signal change for TCL can be computed with Eq. (A4) (see Appendix A) under conditions of $w \rightarrow 0$ and $\tau \rightarrow$ TR. Considering this upper limit as a reference point (i.e., $100 \%$ efficiency), we may now evaluate the efficiency of the quasi-continuous labeling (QCL) experiment applied in the current study. Using the experimental parameters summarized in Appendix A, we obtain an efficiency of $86 \%$. This corresponds roughly to the ratio of the labeling period $\tau$ and TR, which was 0.83 in our study. In terms of sensitivity, the above result might make TCL dispensable. However, in QCL, only the interval $\mathrm{TR}-\tau$ can be used for image acquisition, which restricts the applicability of the method to studies with a relatively small number of slices. Functional perfusion imaging with whole-brain coverage, which is more appropriate for typical cognitive studies, might indeed benefit from a TCL approach. This could be achieved for example by use of a separate labeling gradient as suggested elsewhere (Trampel et al., 2002).

CBF-based studies particularly benefit from a high spatial resolution (Pfeuffer et al., 2002), because partial volume effects reduce the effective percentage signal changes in case of relatively low spatial resolution. $\mathrm{CBF}$ changes of the order of $20 \%$ require the detection of signal changes of less than $0.2 \%$. This is currently below the detection limit of single-subject data, just as it is in conventional BOLD-based fMRI. Therefore, similar to cognitive BOLD studies, which have an even higher contrast-to-noise ratio, multi-subject averaging will be required for cognitive p-fMRI studies. Problems such as a spatial blurring due to the non-perfect registration of single-subject data on a "standard" brain may therefore diminish the effective spatial resolution also in cognitive p-fMRI studies. The extent to which this problem affects the specificity of p-fMRI activations and the optimum spatial resolution for cognitive $\mathrm{p}$-fMRI studies have to be explored in forthcoming studies.

The possibility to quantify $\mathrm{CBF}$ changes is an important advantage of p-fMRI over conventional BOLD-based fMRI. With the presented p-fMRI experiment, quantitative information on $\mathrm{CBF}$ changes was obtained in various cortical areas. The percentage $\mathrm{CBF}$ change during activation is the quotient of the p-fMRI signal change (after removal of residual BOLD contributions) and the CASL signal change in the resting state (as given by the label-map). It should be noted that the $\mathrm{CBF}$ change in a certain brain region obtained by this method is independent from the absolute value of the arterial transit time and also from the amount of intravascular signal contributions in this region. However, due to potential functional changes in the transit times, the $\mathrm{CBF}$ changes reported in Table 1 may be slightly overestimated, cf. Appendix A. In the IFS, task-related $\mathrm{CBF}$ changes have not yet been determined. However, for the primary cortices, such as M1, a selection of data is available. The 26\% CBF change in M1 upon pressing the Yes/No button during the Stroop task can be associated with CBF changes in M1 of the order of $60-100 \%$ observed during more demanding finger-tapping tasks (Hernandez-Garcia et al., 2004; Mildner et al., 2003). Although such differences seem reasonable, more data are required to interpret the $\mathrm{CBF}$ changes as "degree of activation".

\section{Summary}

Functional perfusion imaging by use of CASL at the human common carotid artery was applied to a cognitive paradigm that produces activations in a number of brain regions. CASL was applied quasi-continuously during the functional runs while the label-map necessary for quantification was recorded in a separate short scan. Residual BOLD contributions of the imaging sequence were found to be the main difficulty of the current setup. After the removal of residual BOLD contributions, p-fMRI was capable of reproducing most of the GE-BOLD-fMRI activations as signal changes of opposite sign. The localization of the centers of activations found with p-fMRI agreed reasonably with those from 

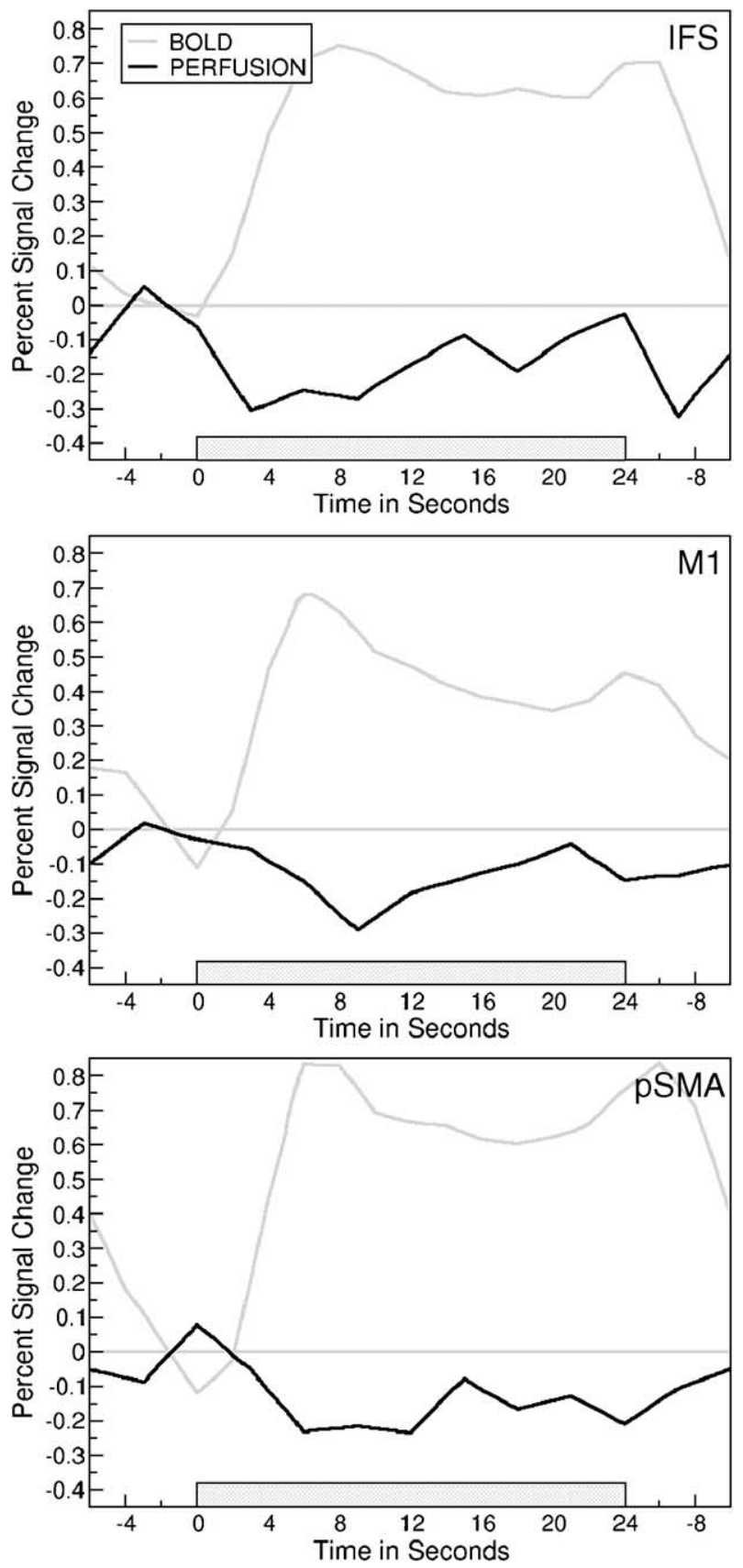

Fig. 3. Time courses of GE-BOLD-fMRI and p-fMRI averaged over a single trial of the Stroop task at the locations as given in Table 1. The shaded area indicates the duration of the stimulus.

GE-BOLD-fMRI. CBF increases during the Stroop task of an order of $20-30 \%$ were obtained.

\section{Acknowledgments}

The authors would like to thank Prof. D. Yves von Cramon for helpful discussions regarding the context of the manuscript. We thank Manfred Weder for technical assistance and Mandy Naumann, Anke Mempel, Simone Wipper, and Annett Wiedemann for experimental support.

\section{Appendix A}

Under the assumption of negligible MT effects (which is fulfilled for the dual-coil setup), the following equation was derived for the CASL signal change in the resting state (Wang et al., 2002):

$$
\begin{aligned}
\frac{\Delta M}{M}_{\text {CASL }}= & -2 \alpha \frac{f}{\lambda} \exp \left(-\delta / T_{1 \mathrm{a}}\right)\left\{T_{1 \mathrm{e}}\left(1-\exp \left((\delta-\tau-w) / T_{1 \mathrm{e}}\right)\right)\right. \\
& \left.+T_{1 \mathrm{a}}\left(\exp \left(\left(\delta-\delta_{\mathrm{a}}\right) / T_{1 \mathrm{a}}\right)-1\right)\right\}
\end{aligned}
$$

with $\frac{1}{T_{1 \mathrm{e}}}=\frac{1}{T_{1}}+\frac{f}{\lambda}$ defining the effective longitudinal

relaxation time.

$T_{1}$ and $T_{1 \mathrm{a}}$ are the longitudinal relaxation times of brain tissue and arterial blood, respectively, $\delta$ is the tissue transit time, $\delta_{\mathrm{a}}$ the arterial transit time, $f$ the blood flow, $w$ the postlabel delay, $\tau$ the length of the labeling period, $\alpha$ the inversion efficiency at the carotid artery, and $\lambda$ the brain-blood partition coefficient. Eq. (A1) is the specific expression of the theory for the conditions $\delta<\tau+w$ and $w<\delta_{\mathrm{a}}$ (Wang et al., 2002). Both conditions are fulfilled for $\mathrm{p}$-fMRI using labeling periods of $\tau=$ $2.5 \mathrm{~s}$ and short post-label delays. In human volunteers, values of $1930 \mathrm{~ms}$ and $1600 \mathrm{~ms}$ were obtained for $\delta$ and $\boldsymbol{\delta}_{\mathrm{a}}$, respectively (Mildner et al., 2005).

For convenience, Eq. (A1) can be expressed by the following formula using the dimensionless quantity $x$ :

$$
\begin{aligned}
\frac{\Delta M}{M}(x)_{\mathrm{CASL}} & =q\left\{\frac{x}{1+x}\left(1-\exp \left(\varphi_{1}(1+x)\right)+g x\right\} \quad\right. \text { with } \\
x & =\frac{T_{1}}{\lambda} f, q=-2 \alpha \exp \left(-\delta / T_{\mathrm{la}}\right), \\
\varphi_{1} & =\frac{\delta-\tau-w}{T_{1}}, \text { and } \\
g & =\frac{T_{1 \mathrm{a}}}{T_{1}}\left(\exp \left(\left(\delta-\delta_{\mathrm{a}}\right) / T_{1 \mathrm{a}}\right)-1\right) .
\end{aligned}
$$

For quasi-continuous labeling and relatively long transit times as present in this work, label from the previous repetition arrives during the next repetition and increases the CASL signal change when compared to a hypothetic experiment with infinite TR. To include this effect in the theory, we assume a $90^{\circ}$ excitation pulse and the conditions $\boldsymbol{\delta}<\boldsymbol{\tau}+w<\mathrm{TR}$ and $\tau+w-\delta>\delta-\boldsymbol{\delta}_{\mathrm{a}}$. Eq. (A3) gives the result for the CASL signal change in the case of finite TR:

$$
\begin{aligned}
& \frac{\Delta M}{M}(x)_{\mathrm{CASL}}=q\left\{\frac { x } { 1 + x } \left(1-\exp \left(\varphi_{1}(1+x)\right)+\exp \left(\varphi_{2}(1+x)\right)\right.\right. \\
& \left.\left.-\exp \left(\varphi_{3}(1+\mathrm{x})\right)\right)+g^{*} x\right\} \\
& \text { with } \varphi_{2}=\frac{\delta-\mathrm{TR}-w}{T_{1}}, \varphi_{3}=\frac{-\mathrm{TR}}{T_{1}} \\
& \text { and } g^{*}=\frac{T_{1 \mathrm{a}}}{T_{1}}\left(\exp \left(\left(\delta-\delta_{\mathrm{a}}\right) / T_{1 \mathrm{a}}\right)-1\right)\left(1-\exp \left(-\mathrm{TR} / T_{1 \mathrm{a}}\right)\right) \text {. }
\end{aligned}
$$


Note that the special case of true-continuous labeling (i.e., labeling is not interrupted for image acquisition) is characterized by the conditions $w \rightarrow 0$ and $\tau \rightarrow \mathrm{TR}$, which yields

$$
\begin{aligned}
& \frac{\Delta M}{M}(x)_{\text {true }-\mathrm{CASL}} \\
& \quad=q\left\{\frac{x}{1+x}\left(1-\exp \left(\varphi_{3}(1+x)\right)\right)+g^{*} x\right\} .
\end{aligned}
$$

An increased CASL signal change of level $\boldsymbol{x}$ can be expanded to first order by a Taylor series around the baseline level $x_{0}$, because $x, x_{0}<<1$ is fulfilled as shown below. This yields

$$
\begin{aligned}
& \frac{\Delta M}{M}(x)_{\mathrm{CASL}}= \frac{\Delta M}{M}\left(x_{0}\right)_{\mathrm{CASL}} \\
&+q_{0}\left\{\begin{array}{c}
\left(1+\sum_{i=1}^{3}(-1)^{i} \exp \left(\varphi_{i, 0}\left(1+x_{0}\right)\right)\left(1+\varphi_{i, 0} x_{0}\right)\right) \\
\left(1+x_{0}\right)^{2}
\end{array} g^{*}+\right. \\
&\left.+\frac{A}{T_{1 \mathrm{a}}}\left\{\begin{array}{c}
\frac{x_{0}}{1+x_{0}}\left(1+\sum_{i=1}^{3}(-1)^{i} \exp \left(\varphi_{i, 0}\left(1+x_{0}\right)\right)\right)+g^{*} x_{0} \\
-\frac{T_{1 \mathrm{a}}}{T_{1}} x_{0}\left(\exp \left(\varphi_{2,0}\left(1+x_{0}\right)\right)-\exp \left(\varphi_{1,0}\left(1+x_{0}\right)\right)\right)
\end{array}\right)\right\} \\
& \times\left(x-x_{0}\right)+O\left(x^{3}\right)
\end{aligned}
$$

Quantities indexed by zero represent their values at baseline level $x_{0} . O\left(x^{3}\right)$ contains all terms of third order in $x$ or $x_{0}$. The quantity $\boldsymbol{A}$ was introduced in order to estimate the influence of transit-time changes and is defined by $\boldsymbol{\delta}-\boldsymbol{\delta}_{0}=-A\left(x-x_{0}\right)$. A linear dependence between the blood flow (and hence, $x$ ) and transit-time changes is probably a simplification but should be justified for small deviations from baseline. The difference between $\delta$ and $\delta_{\text {a }}$ was assumed to stay constant under activation which yields an unchanged factor $g^{*}$.

In the following, we calculate the quotient $Q$ of the p-fMRI signal change and the CASL signal change (label-map). This quotient is given by (Mildner et al., 2003):

$$
Q=\frac{\frac{\Delta M}{M}(x)_{\mathrm{CASL}}-\frac{\Delta M}{M}\left(x_{0}\right)_{\mathrm{CASL}}}{\frac{\Delta M}{M}\left(x_{0}\right)_{\mathrm{CASL}}\left(1+\frac{\Delta M}{M}\left(x_{0}\right)_{\mathrm{CASL}}\right)} .
$$

After insertion of Eqs. (A3) and (A5) into Eq. (A6) and neglection of terms quadratic or higher in $\boldsymbol{x}_{0}$, the following expression is finally obtained:

$$
\begin{aligned}
& Q=\left(\frac{\mathrm{E}+R_{1} x_{0}}{\mathrm{E}+R_{2} x_{0}}\right) \cdot\left[\frac{x-x_{0}}{x_{0}}\right]=D \cdot\left[\frac{x-x_{0}}{x_{0}}\right] \\
& \text { with } E=1+g^{*}+\sum_{i=1}^{3}(-1)^{i} \exp \left(\varphi_{i, 0}\left(1+x_{0}\right)\right), \\
& R_{1}=2 g^{*}+\sum_{i=1}^{3}(-1)^{i} \varphi_{i, 0} \exp \left(\varphi_{i, 0}\left(1+x_{0}\right)\right) \\
& \qquad+\frac{A}{T_{1 \mathrm{a}}}\left\{E-\frac{T_{1 \mathrm{a}}}{T_{1}}\left(\exp \left(\varphi_{2,0}\left(1+x_{0}\right)\right)-\exp \left(\varphi_{1,0}\left(1+x_{0}\right)\right)\right)\right\}
\end{aligned}
$$

and $R_{2}=g^{*}+E+q_{0} E^{2}$.

To obtain the true relative CBF change, $Q$ must be divided by the dimensionless quantity $D$. As an example, we compute this term for a resting CBF level of $73 \mathrm{ml} / \mathrm{min} / 100 \mathrm{~g}$ (Mildner et al., 2005) without and with functional changes in the transit times.
Using values of TR $=3 \mathrm{~s}, T_{1}=1.3 \mathrm{~s}, T_{1 \mathrm{a}}=1.5 \mathrm{~s}, \lambda=0.9 \mathrm{ml} / \mathrm{g}$, $\delta_{0}, \delta_{\mathrm{a}}$, and $\tau$ as above, $\alpha=85 \%$ and $w=250 \mathrm{~ms}$, we obtain $x_{0}=0.018, \varphi_{1,0}=-0.63, \varphi_{2,0}=-1.02, \varphi_{3,0}=-2.31, g^{*}=$ $0.25, q_{0}=-0.47, E=0.98$, and $R_{2}=0.77$. Without functional changes of the transit time $(\boldsymbol{A}=0), R_{1}$ and $D$ have the values 0.68 and 0.998 , respectively. In this case, $Q$ would represent an error of only $-0.2 \%$.

In order to include the effect of changing transit time, the quantity $A$ has to be estimated. For this purpose, we use data from a recent finger-tapping study (Hernandez-Garcia et al., 2004), which reported an arterial transit-time change of $150 \mathrm{~ms}$ and a signal increase of $83 \%$, both averaged over five subjects. The relative blood-flow increase obtained from this signal increase is about $66 \%$. The quantity $A$ can be estimated by $A=150 \mathrm{~ms} / 0.66 / x_{0}=12.9 \mathrm{~s}$. With this value, our above calculation yields $R_{1}=10.82$ and $D=1.18$. Effects from transit-time changes due to activation might therefore lead to an overestimation of the relative $\mathrm{CBF}$ change of the order of $18 \%$.

\section{References}

Alsop, D.C., Detre, J.A., 1996. Reduced transit time sensitivity in noninvasive magnetic resonance imaging of human cerebral blood flow. J. Cereb. Blood Flow Metab. 16, 1236-1249.

Alsop, D.C., Detre, J.A., 1998. Multisection cerebral blood flow MR imaging with continuous arterial spin labeling. Radiology 208, $410-416$

Bosch, V., 2000. Statistical analysis of multi-subject fMRI data: the assessment of focal activations. J. Magn. Reson. Imaging 11, 61-64.

Buxton, R.B., Frank, L.R., Wong, E.C., Siewert, B., Warach, S., Edelman, R.R., 1998. A general kinetic model for quantitative perfusion imaging with arterial spin labeling. Magn. Reson. Med. 40, 383-396.

Calamante, F., Thomas, D.L., Pell, G.S., Wiersma, J., Turner, R., 1999. Measuring cerebral blood flow using magnetic resonance imaging techniques. J. Cereb. Blood Flow Metab. 19, 701-735.

Edelman, R.R., Siewert, B., Darby, D.G., Thangaraj, V., Nobre, A.C., Mesulam, M.M., Warach, S., 1994. Qualitative mapping of cerebral blood flow and functional localization with echo-planar MR imaging and signal targeting with alternating radio frequency. Radiology 192, $513-520$.

Friston, K.J., 1994. Statistical parametric maps in functional imaging: a general linear approach. Hum. Brain Mapp. 2, 189-210.

Friston, K.J., Fletcher, P., Josephs, O., Holmes, A., Rugg, M.D., Turner, R., 1998. Event-related fMRI: characterizing differential responses. NeuroImage 7, 30-40.

Gonzalez-At, J.B., Alsop, D.C., Detre, J.A., 2000. Cerebral perfusion and arterial transit time changes during task activation determined with continuous arterial spin labeling. Magn. Reson. Med. 43, 739-746.

Hernandez-Garcia, L., Lee, G.R., Vazquez, A.L., Noll, D.C., 2004. Fast, pseudo-continuous arterial spin labeling for functional imaging using a two-coil system. Magn. Reson. Med. 51, 577-585.

Jesmanowicz, A., Bandettini, P.A., Hyde, J.S., 1998. Single-shot half $k$ space high-resolution gradient-recalled EPI for fMRI at 3 Tesla. Magn. Reson. Med. 40, 754-762.

Kwong, K.K., Belliveau, J.W., Chesler, D.A., Goldberg, I.E., Weisskoff, R.M., Poncelet, B.P., Kennedy, D.N., Hoppell, B.E., Cohen, M.S., Turner, R., Cheng, H.M., Brady, T.J., Rosen, B.R., 1992. Dynamic magnetic resonance imaging of human brain activity during primary sensory stimulation. Proc. Natl. Acad. Sci. U. S. A. 89, 5675-5679.

Lohmann, G., Mueller, K., Bosch, V., Mentzel, H., Hessler, S., Chen, L., Zysset, S., von Cramon, D.Y., 2001. Lipsia-A new software system for the evaluation of functional magnetic resonance images of the human brain. Comput. Med. Imaging Graph 25, 449-457. 
Merkle, H., Driesel, W., 2003. 3 Tesla general purpose transceive helmet coil. MAGMA 16 (Suppl. 7), S87.

Mildner, T., Trampel, R., Möller, H.E., Schaefer, A., Wiggins, C.J., Norris, D.G., 2003. Functional perfusion imaging using continuous arterial spin labeling with separate labeling and imaging coils at 3 T. Magn. Reson. Med. 49, 791-795.

Mildner, T., Möller, H.E., Driesel, W., Norris, D.G., Trampel, R., 2005. Continuous arterial spin labeling at the human common carotid artery: the influence of transit times. NMR Biomed. 18, 19-23.

Möller, H.E., Klocke, H., Bongartz, G.M., Peters, P.E., 1996. MR flow quantification using RACE: clinical application to the carotid arteries. J. Magn. Reson. Imaging 6, 503-512.

Mugler, J.P., Brookeman, J.R., 1990. Three-dimensional magnetizationprepared rapid gradient-echo imaging (3D MP RAGE). Magn. Reson. Med. 15, 152-157.

Norris, D.G., 2000. Reduced power multislice MDEFT imaging. J. Magn. Reson. Imaging 11, 445-451.

Norris, D.G., Zysset, S., Mildner, T., Wiggins, C.J., 2002. An investigation of the value of spin-echo-based fMRI using a Stroop color word matching task and EPI at 3 T. NeuroImage 15, 719-726.

Pekar, J., Jezzard, P., Roberts, D.A., Leigh, J.S., Frank, J.A., McLaughlin, A.C., 1996. Perfusion imaging with compensation for asymmetric magnetization transfer effects. Magn. Reson. Med. 35, 70-79.

Pfeuffer, J., Adriany, G., Shmuel, A., Yacoub, E., VanDeMoortele, P., Hu, X., Ugurbil, K., 2002. Perfusion-based high-resolution functional imaging in the human brain at 7 Tesla. Magn. Reson. Med. 47, 903-911.

Siewert, B., Bly, B.M., Schlaug, G., Darby, D.G., Thangaraj, V., Warach, S., Edelman, R.R., 1996. Comparison of the BOLD- and EPISTARtechnique for functional brain imaging by using signal detection theory. Magn. Reson. Med. 36, 249-255.

Stroop, J., 1935. Studies of interference in serial verbal reactions. J. Exp. Psychol. 18, 643-662.

Talagala, S.L., Ye, F.Q., Ledden, P.J., Chesnick, S., 2004. Whole-brain 3D perfusion MRI at 3.0 T using CASL with a separate labeling coil. Magn. Reson. Med. 52, 131-140.

Talairach, P., Tournox, J., 1988. A Stereotactic Coplanar Atlas of the Human Brain. Thieme, Stuttgart.

Trampel, R., Mildner, T., Goerke, U., Schaefer, A., Driesel, W., Norris, D.G., 2002. Continuous arterial spin labeling using a local magnetic field gradient coil. Magn. Reson. Med. 48, 543-546.
Trampel, R., Jochimsen, T.H., Mildner, T., Norris, D.G., Möller, H.E., 2004. Efficiency of flow-driven adiabatic spin inversion under realistic experimental conditions: a computer simulation. Magn. Reson. Med. 51, 1187-1193.

Ugurbil, K., Ogawa, S., Kim, S.G., Hu, X., Chen, W., Zhu, X.H., 1999. Imaging brain activity using nuclear spins. In: Maraviglia, B. (Ed.), Proc. of the International School of Physics "Enrico Fermi", Magnetic Resonance and Brain Function: Approaches from Physics. Elsevier, Amsterdam, pp. 261-301.

Wang, J., Alsop, D.C., Li, L., Listerud, J., Gonzalez-At, J.B., Schnall, M.D., Detre, J.A., 2002. Comparison of quantitative perfusion imaging using arterial spin labeling at 1.5 and 4.0 Tesla. Magn. Reson. Med. 48, 242-254.

Wang, J., Aguirre, G.K., Kimberg, D.Y., Roc, A.C., Li, L., Detre, J.A., 2003. Arterial spin labeling perfusion fMRI with very low task frequency. Magn. Reson. Med. 49, 796-802.

Worsley, K., Friston, K.J., 1995. Analysis of fMRI time-series revisitedAgain. NeuroImage 2, 173-181.

Yang, Y., Frank, J.A., Hou, L., Ye, F.Q., McLaughlin, A.C., Duyn, J.H., 1998. Multislice imaging of quantitative cerebral perfusion with pulsed arterial spin labeling. Magn. Reson. Med. 39, 825-832.

Ye, F.Q., Smith, A.M., Yang, Y., Duyn, J.H., Mattay, V.S., Ruttimann, U.E., Frank, J.A., Weinberger, D.R., McLaughlin, A.C., 1997. Quantitation of regional cerebral blood flow increases during motor activation: a steady-state arterial spin tagging study. NeuroImage 6, 104-112.

Ye, F.Q., Smith, A.M., Mattay, V.S., Ruttimann, U.E., Frank, J.A., Weinberger, D.R., McLaughlin, A.C., 1998. Quantitation of regional cerebral blood flow increases in prefrontal cortex during a working memory task: a steady-state arterial spin-tagging study. NeuroImage 8 , 44-49.

Zaharchuk, G., Ledden, P.J., Kwong, K.K., Reese, T.G., Rosen, B.R., Wald, L.L., 1999. Multislice perfusion and perfusion territory imaging in humans with separate label and image coils. Magn. Reson. Med. 41, 1093-1098.

Zhang, W., Silva, A.C., Williams, D.S., Koretsky, A.P., 1995. NMR measurement of perfusion using arterial spin labeling without saturation of macromolecular spins. Magn. Reson. Med. 33, 370-376.

Zysset, S., Müller, K., Lohmann, G., von Cramon, D.Y., 2001. Color-word matching Stroop task: separating interference and response conflict. NeuroImage 13, 29-36. 